\title{
Efetividade de um programa fisioterapêutico proprioceptivo para treino de equilíbrio em idosos institucionalizados
}

\author{
Gabriela Guimarães Oliveira*, Fernando Antônio De Mello Prati*
}

\section{Resumo}

O objetivo do presente estudo foi avaliar os efeitos de um Programa Fisioterapêutico Proprioceptivo para treino de equilíbrio em idosos institucionalizados, levando em consideração a amostra geral e os gêneros. Participaram 14 idosos (sete homens e sete mulheres) de ambos os gêneros. A coleta de dados iniciou-se, primeiramente, através de uma revisão de prontuários, a fim de analisar o histórico de saúde dos possíveis voluntários. Foram incluídos os idosos que se enquadravam nos três critérios a seguir: deambulavam sem auxílio; não apresentavam evidências de limitação funcional, ao serem submetidos à aplicação do Índice de Katz e compreendiam comandos verbais, não apresentando evidências de limitação cognitiva, ao serem submetidos à aplicação do Mini Exame do Estado Mental. Por outro lado, foram excluídos os portadores de doenças crônicas ou agudas que apresentavam comprometimento físico e/ou funcionais que limitassem a realização dos exercícios propostos ou que, durante a intervenção,totalizassem mais de três faltas. Esses foram avaliados primeiramente pela Escala de Equilíbrio de Berg e, posteriormente, foram submetidos a um programa de exercícios fisioterapêuticos baseados em atividades para facilitação do equilíbrio poe meio do trabalho de propriocepção. A intervenção constou de 12 sessões, com uma frequência de duas sessões por semana, durante cinquenta minutos, totalizando seis semanas (aproximadamente um mês e meio) e em grupo de no máximo três idosos. Cada voluntário esteve acompanhado em todos os exercícios por um pesquisador ou estagiário. Após esse período, os idosos foram reavaliados pelo mesmo instrumento. $\mathrm{O}$ teste de $\mathrm{t}$ de Student para amostras pareadas foi utilizado para comparação pré e pós-intervenção e para medidas independentes para comparar os gêneros. Adotou-se um nível de significância de $p \geq 0,05$. De forma geral, os idosos apresentaram um aumento significativo nos valores da escala no momento pós-intervenção $(p<0,001)$. Não houve diferença significativa entre os gêneros, no momento pré $(p=0,699)$ e pós-intervenção $(p=0,888)$. A partir dos resultados encontrados neste estudo, pode-se concluir que esse Programa Fisioterapêutico Proprioceptivo específico aplicado nesse grupo possibilitou melhora significativa no escore total da Escala de Equilíbrio de Berg para a amostra total, como de ambos os gêneros isoladamente. No entanto, não apresentou diferença significativa entre os gêneros, o que nos leva a crer que o nível de atividade física e o baixo risco de queda encontrado na população estudada, em ambos os gêneros, possa ter interferido nesse quesito.

Palavras-chave: Equilíbrio postural. Idosos. Fisioterapia.

* Acadêmica do Curso de Fisioterapia do Centro Universitário Metodista do IPA. Endereço: Av. Heitor Vieira, 1920 Belém Novo, Porto Alegre (RS), CEP: 91780-000. E-mail: oliveira_gabriela@hotmail.com.

** Fisioterapeuta, Mestre e Docente do Curso de Fisioterapia do Centro Universitário Metodista do IPA, Porto Alegre (RS). E-mail: fernando.prati@metodistadosul.edu.br.

$\rightarrow$ http://dx.doi.org/10.5335/rbceh.2014.3402

Recebido em: 10/08/2013. Aprovado em: 01/08/2014 


\section{Introdução}

A população brasileira vem sofrendo, nas últimas cinco décadas, transições decorrentes de mudanças nos níveis de mortalidade e de fecundidade, em ritmos nunca vistos anteriormente. Nesse contexto, a população idosa vem se destacando por apresentar um crescimento significativo. Hoje, ela representa quase 18 milhões de pessoas, constituindo $12 \%$ da população total e estima-se que, no ano de 2050, um quinto da população brasileira seja constituída por idosos (IBGE, 2011).

Assim, o processo de envelhecimento é uma temática que vem sendo abordada constantemente com o passar do tempo (BECHARA; SANTOS, 2008). Esse processo manifesta-se por declínio das funções dos diversos órgãos e sistemas, devido ao desgaste natural do corpo que faz com que o organismo comece a apresentar falhas diante das funções primordiais de manutenção corporal (PAULA, 2010).

Dessa forma, para que o equilíbrio corporal ocorra de forma adequada é necessário um conjunto de estruturas funcionalmente entrosadas, são elas: o sistema vestibular, a visão e o sistema somatossensorial (propriocepção). Dentre esse conjunto de estruturas, a propriocepção enquadra-se como a capacidade perceptiva motora mais importante para manter o equilíbrio e a funcionalidade (FREITAS; CARVALHO; VILAS BOAS, 2013).

A propriocepção é uma variação especializada da modalidade sensitiva do tato. De modo específico, a propriocepção corresponde a um mecanismo de percepção que envia uma informação para $o$ Sistema Nervoso Central (via aferentes) a partir de mecanorreceptores existentes nas cápsulas articulares, ligamentos, músculos, tendões e pele, que, após serem interpretadas são reenviadas (vias eferentes) para o corpo a fim de manter o controle postural (ELLENBECKER, 2002). Dessa forma, o comprometimento estrutural desse sistema gera um dos principais fatores que, hoje, limita a vida do idoso, a instabilidade postural, que vem acompanhada da dificuldade em manter posturas adequadas e que frequentemente ocasionam as quedas (ESQUENAZI; SILVA; GUIMARÃES, 2014; SOUTHIARD et al., 2005).

A queda é considerada um evento crítico na vida desses indivíduos, sendo o acidente mais frequente entre eles. No Brasil, cerca de $30 \%$ dos idosos caem uma vez ao ano. A queda pode gerar desde fraturas até limitações, imobilidade e isolamento social. Cerca de $5 \%$ a $10 \%$ das quedas resultam em ferimentos importantes. Em razão das suas complicações, é também considerada a principal causa de morte naqueles com idade superior a 65 anos, pois seu risco é aumentado com o avanço da idade, podendo atingir 51\% dos idosos acima de 85 anos. Nessa faixa etária, elas estão intimamente relacionadas com a marcha e a postura (MACIEL; GUERRA, 2005; FRANCIULLI et al., 2007; CUNHA; LOURENÇO, 2014).

Nesse aspecto, com a perda esperada de propriocepção durante o envelhecimento, aumenta-se o limiar para a detecção do movimento articular, ou seja, ocorre o retardo das respostas posturais 
automáticas, que contribui para a ocorrência de quedas (COSTA et. al., 2013). Assim, tal sistema necessita ser estimulado com certa frequência. Figueiredo et al. (2011) confirmaram um maior nível de comprometimento de equilíbrio funcional em grupo de idosos quando comparados aosadolescentes, jovens e adultos e comprovam que indivíduos participantes de atividades multissensoriais com enfoque na estimulação proprioceptiva demonstraram maior estabilidade postural quando comparados a um grupo controle.

Paralelamente a isso, para Borges et al. (2013), idosos moradores de instituições de longa permanência são ainda mais vulneráveis a apresentar fragilidade, declínio funcional e alterações no sistema proprioceptivo quando comparados a idosos não institucionalizados, ao passo que alterações esperadas devido ao processo de envelhecimento podem ser agravadas pelas dificuldades de adaptação dos idosos às novas condições de vida, e pela falta de motivação e encorajamento comuns nesses ambientes.

Portanto, considerando o crescente aumento da população idosa e o envelhecimento como um acontecimento inevitável, acompanhado de declínio funcional, com consequentes alterações posturais de equilíbrio e de episódios de queda, este estudo tem por objetivo avaliar os efeitos de um Programa Fisioterapêutico Proprioceptivo para treino de equilíbrio em idosos institucionalizados, levando em consideração a amostra geral e os gêneros.

Apontamos como hipóteses, que esse Programa Fisioterapêutico Proprioceptivo específico possibilitará melhora significativa no escore total da Escala de Equilíbrio de Berg para a amostra total. Assim como, apresentará diferença significativa no escore entre os gêneros.

\section{Materiais e métodos}

Este estudo foi revisado e aprovado em 9 de dezembro de 2011 sob protocolo n. 333 pelo Comitê de Ética em Pesquisa do Centro Universitário Metodista do Instituto Porto Alegre (IPA), conforme previsto na resolução 196/96 do Conselho Nacional de Saúde - CNS. Realizou-se um estudo de caráter quase experimental, cuja amostra foi selecionada na instituição asilar Padre Cacique, Porto Alegre - RS.

Depois de esclarecidos verbalmente sobre os procedimentos aos quais seriam submetidos, os idosos que concordaram, assinaram um termo de consentimento livre e esclarecido (TCLE). No caso de analfabetos ou impossibilitados, o termo foi assinado pelo Fisioterapeuta da instituição, com a concordância do voluntário. Da mesma forma, foi firmado com a instituição em questão um termo de autorização institucional para a realização desta pesquisa.

A coleta de dados ocorreu nas dependências do Asilo Padre Cacique, primeiramente, por meio de uma revisão de prontuários, a fim de analisar o histórico de saúde dos possíveis voluntários. Foram incluídos no estudo, os idosos que se enquadravam nos três critérios a seguir: os que deambulavam sem auxílio; os que não apresentavam evidências de limitação funcional após serem submetidos à aplicação do Índice de Katz (LINO et 
al., 2008; KATZ, 1976) e os que compreendiam comandos verbais, não apresentando evidências de limitação cognitiva, após serem submetidos à aplicação do Mini Exame do Estado mental (MEEM) (OLIVEIRA; GORETTI; PEREIRA, 2006; FOLSTEIN; FOLSTEIN; MCHUGH, 1975; BERTOLUCCI et al., 1994). Foram excluídos do estudo os portadores de doenças crônicas ou agudas que apresentavam comprometimento físico e/ou funcionais que limitassem a realização dos exercícios propostos ou que totalizaram mais de três faltas na intervenção.

Os idosos tiveram, inicialmente, seu equilíbrio avaliado pela Escala de Equilíbrio de Berg. Essa escala avalia o equilíbrio do indivíduo em 14 situações, representativas de atividades do dia a dia, tais como: ficar de pé, levantar-se, andar, inclinar-se à frente, transferir-se, virar-se, dentre outras. A pontuação máxima a ser alcançada é de 56 pontos e cada item possui uma escala ordinal de cinco alternativas, variando de 0 a 4 pontos, de acordo com o grau de dificuldade (MIYAMOTO et al., 2004; WHITNEY; WRISLEY; FURMAN, 2003).

Posteriormente, os idosos foram submetidos a um programa de exercícios fisioterapêuticos baseados em atividades para facilitação do equilíbrio por meio do trabalho de propriocepção. A intervenção foi realizada em 12 sessões de cinquenta minutos, duas vezes por semana, durante cinquenta minutos, totalizando seis semanas (aproximadamente um mês e meio) e em grupo de no máximo três idosos. Cada voluntário esteve acompanhado em todos os exercícios por um pesquisador e/ou estagiários, que foram devidamente treinados para a aplicação dos exercícios. A pesquisa ocorreu no período de 9 de janeiro de 2012 a 24 de fevereiro de 2012 na sala de atendimentos fisioterapêuticos da instituição, um local amplo e propício para a realização das atividades propostas, além de representar um local de fácil acesso para os voluntários.

No início e no final de cada procedimento, foi realizada a verificação dos sinais vitais (frequência cardíaca, frequência respiratória e pressão arterial), a fim de controlar possíveis alterações desses sinais durante os treinos. Em seguida, era realizada uma forma individual de alongamento de membro superior (peitorais, tríceps braquial e deltoide porção média) e inferior (quadríceps, isquiotibiais e tríceps sural) durante 30 segundos em cada membro (LOPES; PASSERINI; TRAVENSOLO, 2010), assim como uma forma de aquecimento (bicicleta ou esteira ergométrica) que também foi realizada no início, durante cinco minutos (BECHARA; SANTOS, 2008). Logo, foi aplicado um circuito de quatro exercícios, em que três eram específicos para equilíbrio e um para marcha. $\mathrm{O}$ circuito durava em média vinte minutos, dependendo da facilidade e da compressão do voluntário na realização dos exercícios, assim como do ritmo adotado por ele. Todos os exercícios eram realizados em todos os dias de treino e seguiam sempre a sequência a seguir. 1) No primeiro exercício, o idoso permanecia sentado na bola suíça em apoio bipodal com os braços entrelaçados e realizava movimentos de ula-ula (movimento de anteversão e retroversão pélvica e incli- 
nação lateral pélvica direita e esquerda), num total de oito movimentos para cada direção (SOUZA, 2004). 2) No segundo exercício, o idoso permanecia sentado na bola suíça, com apoio unipodal e abdução de $90^{\circ}$ do ombro contralateral, onde sustentava uma bola pequena e leve na mão por dez segundos e, então, invertia o membro de apoio. Caso o voluntário demonstrasse facilidade, era associada a rotação de tronco. $\mathrm{O}$ exercício era bilateral, num total de oito repetições para cada lado (BECHARA; SANTOS, 2008). 3) No terceiro exercício, o idoso permanecia em apoio bipodal, porém em um plano instável (cama elástica). Ele realizava arremessos de uma bola pequena e leve, assim como a recebia da pesquisadora. Esse exercício durou cinco minutos (SOUZA, 2004). 4) No quarto exercício, o idoso realizava uma caminhada em linha reta sobre linha demarcada em uma superfície instável (colchonete). $\mathrm{O}$ voluntário era estimulado a realizar o contato inicial com o solo no sentido calcâneo - hálux, primeiramente com os olhos abertos (dois minutos) e posteriormente com a adição de pequenos cones como obstáculo (dois minutos). Caso ele demonstrasse facilidade, a caminhada calcâneo - hálux era realizada de olhos fechados sobre a linha demarcada (um minuto). Esse exercício durou no máximo cinco minutos (SOUZA, 2004).

Após o período de intervenção, os idosos foram reavaliados pelo mesmo instrumento.

Nossa amostra inicial era composta por 18 idosos (dez homens e oito mulheres). Ao término contávamos com 14 desses (sete homens e sete mulheres).
Houve duas perdas por desistência (dois homens) e duas perdas por não atingirem o escore mínimo no Mini Exame do Estado Mental (uma mulher e um homem). A amostra era composta por idosos fisicamente ativos, classificados em baixo risco de queda. Todos os idosos praticavam desde uma simples caminhada diária por todas as dependências do asilo, até atividades físicas promovidas pela própria instituição. Além disso, grande parte realizava atendimentos fisioterapêuticos, com uma frequência de no mínimo duas vezes por semana. No entanto, a coleta de dados se deu em período de férias, no qual os idosos não estavam realizando esses atendimentos.

Para verificar a normalidade do conjunto de dados foi feito o teste de Shapiro-Wilk. Todos os dados contínuos foram descritos por média e desvio-padrão. Dos resultados avaliados pela escala de Berg, pré e pós-intervenção, foi realizado o teste de $t$ de Student para amostras pareadas. O teste $t$ de Student para medidas independentes foi utilizado para comparar os gêneros. Todos os dados foram armazenados e analisados no software Statistical Package for the Social Sciences for Windows (SPSS) versão 20.0, sendo adotado um nível de significância de $\mathrm{p} \leq 0,05$.

\section{Resultados}

Na Tabela 1, estão descritas as variáveis referentes à caracterização da amostra estudada. 
Tabela 1 - Caracterização da amostra

\begin{tabular}{|c|c|}
\hline Variáveis & $n=14$ \\
\hline Idade (anos) - Média \pm DP [mín - máx] & $76,64 \pm 10,63[63-94]$ \\
\hline \multicolumn{2}{|l|}{ Sexo $-n(\%)$} \\
\hline Masculino & $7(50)$ \\
\hline Feminino & $7(50)$ \\
\hline \multicolumn{2}{|l|}{ Escore de Berg - Média \pm DP (mín- máx) } \\
\hline Pré-Intervenção & $48,36 \pm 4,57(0-56)$ \\
\hline Pós-Intervenção & $50,57 \pm 3,57(0-56)$ \\
\hline
\end{tabular}

Nota: Idade em anos; DP=desvio padrão; $n=$ número de indivíduo; \%=porcentagem; min=mínimo; máx=máximo

Comparando o escore de Berg pré e pós-intervenção, levando-se em consideração a amostra geral, os idosos tiveram uma melhora estatisticamente significativa $(\mathrm{p}<0,001)$.

Levando em consideração os gêneros, os homens obtiveram um incremento de
1,85 pontos no escore. Essa diferença foi estatisticamente significativa ( $\mathrm{p}=0,007)$. As mulheres obtiveram um incremento de 2,57 pontos no escore. Essa diferença também foi estatisticamente significativa $(\mathrm{p}<0,001)$ (Tabela 2$)$.

Tabela 2 - Diferenças e médias entre homens e mulheres na pré e pós-intervenção

\begin{tabular}{l|c|c|c|c}
\cline { 2 - 5 } & Idade & Pré-Berg & Pós-Berg & Diferença \\
\cline { 2 - 5 } & Média \pm DP & $\begin{array}{c}\text { Média } \pm \text { DP } \\
\text { (mín - máx) }\end{array}$ & $\begin{array}{c}\text { Média } \pm \text { DP } \\
\text { (mín - máx) }\end{array}$ & Pré/Pós (p) \\
\hline Total (14) & $76,64 \pm 10,63$ & $\begin{array}{c}48,36 \pm 4,57 \\
(0-56)\end{array}$ & $\begin{array}{c}50,57 \pm 3,57 \\
(0-56)\end{array}$ & $<0,001^{*}$ \\
Homens (7) & $84,00 \pm 7,87$ & $\begin{array}{c}48,86 \pm 3,71 \\
(0-56)\end{array}$ & $\begin{array}{c}50,71 \pm 3,09 \\
(0-56)\end{array}$ & $0,007^{*}$ \\
Mulheres (7) & $69,29 \pm 7,52$ & $\begin{array}{c}47,86 \pm 5,55 \\
(0-56)\end{array}$ & $\begin{array}{c}50,43 \pm 4,23 \\
(0-56)\end{array}$ & $<0,001^{*}$ \\
\hline
\end{tabular}

Nota: Idade em anos, DP=desvio padrão, p=significância, * = estatisticamente significativo.

Mesmo com as diferenças numéricas de escore no momento pré em relação aos gêneros, e um incremento maior para mulheres na pós-intervenção, não houve diferença significativa entre homens e mulheres, em relação ao momento pré $(p=0,699)$ e momento pós $(p=0,888)$ inter- venção. No entanto, a comparação entre a média de idade dos homens e das mulheres foi considerada estatisticamente significativa $(\mathrm{p}=0,004)$.

De acordo com os valores no momento pré-intervenção, todos os idosos já se encontravam em baixo risco de queda, no 
entanto os valores de todos os idosos na pós-intervenção aumentaram ou então se mantiveram iguais.

\section{Discussão}

A população idosa é frequentemente utilizada como objeto de estudo em trabalhos que procuram verificar a efetividade de exercícios para melhora do equilíbrio, uma vez que os déficits de equilíbrio constituem um fator de risco que pode ser modificável por meio de uma intervenção baseada em exercícios (LUNDEBJERG, 2001). Portanto, muitos estudos têm sido direcionados à efetividade de intervenções sobre o treino de equilíbrio, reduzindo o risco de quedas em idosos (BECHARA; SANTOS, 2008; LOPES; PASSERINI; TRAVENSOLO, 2010; ZAMBALDI et al., 2007; NASCIMENTO; PATRIZZI; OLIVEIRA, 2012). Entretanto, Gardner et al. (2000) apontam que a maioria desses estudos aborda populações não institucionalizadas. Assim, optamos por propor um programa voltado para o trabalho de propriocepção em idosos institucionalizados, pois esses caem em média três vezes mais do que os que vivem em seus lares (AIKAWA; BRACCIALLI; PADULA, 2006).

Gauchard et al. (2003) demonstraram que uma intervenção de exercícios com ênfase na propriocepção, traz melhoras significativas nos padrões de equilíbrio postural em relação a exercícios somente de caminhada e corrida. Da mesma forma, Lanuez e Jacob Filho (2008) também relataram que a melhora do equilíbrio em seu grupo praticante de exercícios aeróbios deve-se ao fato das caminhadas terem sido realizadas em terrenos irregulares, ocorrendo, assim, estímulos em diversos sistemas responsáveis pelo controle postural. Estudos que nos fizeram inserir o trabalho de propriocepção como foco deste trabalho.

Nascimento et al. (2012) estudaram o efeito do treinamento proprioceptivo nas medidas do equilíbrio postural de idosos e observaram um aumento significativo no escore total da Escala de equilíbrio de Berg de 50,8 para 53,6 após a intervenção. Bechara e Santos (2008) analisaram a efetividade de um programa fisioterapêutico para treino de equilíbrio em idosos e obtiveram valores significativos na Escala de Equilíbrio de Berg de 49,7 na pré-intervenção para 55,5 na pós-intervenção. Valores semelhantes foram encontrados no atual estudo, no qual a amostra geral de idosos apresentou um aumento no escore total de 48,36 para 50,57, demonstrando significativa melhora na condição de equilíbrio dinâmico e estático.

Neste estudo, os homens tiveram um incremento de 1,85 pontos no escore da Escala de Berg e as mulheres tiveram um incremento de 2,57 pontos no escore da Escala de Berg pós-intervenção, evento que foi considerado estatisticamente significativo e que provavelmente ocorreu devido à diferença significativa na média de idade entre os sexos, que foi de 15 anos. Tal resultado vai ao encontro do estudo de Steadman et al. (2003), que tiveram como objetivo avaliar a eficácia de um programa de treinamento de equilíbrio na melhoria da mobilidade e do bem estar de pessoas idosas com problemas de equilíbrio por meio da Escala 
de Equilíbrio de Berg e verificaram que as mulheres tiveram uma melhora um pouco superior a dos homens.

Em relação aos valores de pré e pós-intervenção entre homens e mulheres, não houve diferença significativa. $O$ que vai ao encontro do estudo de Yokokawa et al. (2003) que analisaram o equilíbrio de idosos institucionalizados pré e pós-programa de exercícios fisioterapêuticos e os submeteram à escala de Equilíbrio de Berg e verificaram que não houve diferença no escore da escala pré e pós-intervenção entre homens e mulheres.

Em nosso estudo, observou-se que a relação entre a média de idade de homens e mulheres foi considerado estatisticamente significativo. No entanto, isso não interferiu nos valores pré e pós-intervenção. Esses resultados vão contra o estudo de Dias et al. (2009) que analisaram o déficit de equilíbrio de idosos em diferentes faixas etárias e detectaram que no grupo etário acima dos 80 anos, o equilíbrio foi inferior, com diferença significativa, em relação aos idosos mais novos. Porém, acreditamos que tal fato ocorreu porque a maioria dos idosos participantes do estudo, independente do sexo, praticava alguma atividade física na instituição asilar, assim como recebia atendimentos fisioterapêuticos. Dessa forma, apontamos que esses resultados devem-se à presença da atividade física na vida desses indivíduos. O que vai ao encontro do estudo de Silva et al. (2010) que estudaram o desempenho de idosos que praticavam atividade física regularmente e sedentários, observando que os idosos sedentários tinham maior comprometimento do equilíbrio e, consequentemente, maior ocorrência de quedas. Da mesma forma, como Figliolino et al. (2009) que compararam idosos praticantes e não praticantes de atividade física, em relação à probabilidade de quedas, desequilíbrio e dificuldades na realização das atividades básicas e instrumentais de vida diária e puderam observar que os idosos praticantes de atividade física apresentaram melhores resultados nesses quesitos.

Por fim, esses achados evidenciam a importância da inserção de treinamentos que busquem a melhora do equilíbrio da população idosa, uma vez que tal grupo pode ser beneficiado pelo programa de treinamento proprioceptivo, minimizando os efeitos fisiológicos do envelhecimento.

Assim, apontamos como limitação deste estudo o baixo tamanho amostral, que dificulta a aplicação de análises de correlação, sobretudo quando estratificamos o grupo em gênero e comparamos faixa etária. Apontamos também, a não homogeneidade do grupo em relação à idade, ao passo que diferenças discrepantes, principalmente na faixa etária idosa, podem vir a influenciar de forma significativa uma amostra. Além disso, o fato dos idosos já estarem inseridos na classificação de baixo risco de queda, o que não nos possibilitou observarmos melhoras tão significativas.

\section{Conclusão}

A partir dos resultados encontrados neste estudo, pode-se concluir que este Programa Fisioterapêutico Proprioceptivo específico aplicado nesse grupo pos- 
sibilitou melhora significativa no escore total da Escala de Equilíbrio de Berg para a amostra total, como de ambos os gêneros isoladamente. No entanto, não acarretou diferença significativa entre os gêneros, o que refuta uma de nossas hipóteses e nos leva a crer que o nível de atividade física e o baixo risco de queda encontrada na população estudada, em ambos os gêneros, possa ter interferido nesse quesito.

Sugerimos que novas pesquisas que confirmem a melhora do equilíbrio e a diminuição de possibilidade de quedas em idosos sejam realizadas e que essas envolvam uma amostragem mais significativa, façam uso de maior tempo de intervenção e utilizem uma população com maior comprometimento de equilíbrio.

Logo, espera-se que esta pesquisa possa contribuir para estudos futuros sobre o equilíbrio da população idosa.

\section{Effectiveness of a proprioceptive physiotherapeutic program for balance training in institucionalized elderly}

\section{Abstract}

The objective of this study was to evaluate the effects of a Proprioceptive Physiotherapeutic Program for balance training in institutionalized elderly, taking into account the overall sample and genres. Participated 14 elderly of both genres (seven men and seven women). Data collection was initiated primarily through a review of medical records in order to analyze the health history of potential volunteers. Elderly who fit were included the three following criteria: ambulating without assistance; with no evidence of functional limitation, when subjected to the application of the Katz Index and understand verbal commands, showing no evidence of cognitive limitation, when subjected to the application of the Mini Mental State Examination. On the other hand, were excluded elderly with diseases chronic or acute presenting physical and / or functional impairment that limited the performance of the proposed exercise or during the intervention had more than three absences. These were first assessed by the Berg Balance Scale and subsequently subjected to a program of physical therapy exercises based on activities to facilitate the balance through the work of proprioception. The intervention consisted of 12 sessions, with a frequency of two sessions per week for 50 minutes, totaling six weeks (about a month and a half) and in groups of up to three elderly. Each volunteer was accompanied in all exercises by a researcher, and/or trainees. After this period, they were reevaluated by the same instrument. The $t$ test for paired samples was used to compare pre -and post- intervention and independent measures to compare the genders. We adopted a significance level of $p \geq 0.05$. In general, the elderly showed a significant increase in the scale values in the post-intervention $(p<0.001)$. There was no significant difference between genders in the pre $(p=0.699)$ and post - intervention $(p=0.888)$. Based on the results of this study, it can be concluded that this specific Physiotherapeutic Proprioceptive program applied in this group enabled a significant improvement in the total score of the Berg Balance Scale for the total sample, as both genders separately. However, not resulted a significant difference between genders, which leads us to believe that the level of physical activity and low downside risk found in the study population, in both genders, may have interfered in this regard.

Keywords: Postural Balance. Elderly. Physical Therapy. 


\section{Referências}

AIKAWA, A. C.; BRACCIALLI, L. M. P.; PADULA, R. S. Efeitos das alterações posturais e de equilíbrio estático nas quedas de idosos institucionalizados. Rev. Ciênc. Méd., Campinas-SP, v. 15, n. 3, p. 189-196, maio/ jun. 2006.

BECHARA, F. T.; SANTOS, S. M. S. Efetividade de um programa fisioterapêutico para treino de equilíbrio em idosos. Revista Saúde e Pesquisa, Maringá, v. 1, n. 1, p. 15-20, jan./ abr. 2008.

BERTOLUCCI, P. H. F.; BRUCKI, S. M. D.; CAMPACCI, S. R.; JULIANO, Y. O Mini-exame do Estado Mental em uma população geral. Impacto da escolaridade. Arq. Neuropsiquiatr., São Paulo, v. 52, n. 1, p. 1-7, mar. 1994.

BORGES, C. L.; SILVA, M. J.; CLARES, J. W.B.; BESSA, M. E. P.; FREITAS, M. C. P. Avaliação da fragilidade de idosos institucionalizados. Acta Paul. Enferm., São Paulo, v. 26, n. 4, p. 318-322, out./dez. 2013.

IBGE - Instituto Brasileiro de Geografia Estatística. Indicadores sociais municipais - Uma Análise dos Resultados do Universo do Censo Demográfico 2010. Estudos \& Pesquisas: Informação Demográfica e Socioeconômica, Rio de Janeiro, n. 28, 2011, 149 p.

COSTA, A. G. S.; ARAUJO, T. L.; OLIVEIRA, A. R. S.; MORAIS, H. C. C.; SILVA, V. M.; LOPES, M. V. O. Fatores de risco para quedas em idosos. Rev. Rene, Fortaleza, v. 14, n. 4 , p. 821-828, 2013.

CUNHA, A.; LOURENÇO, R. Quedas em idosos: prevalência e fatores associados. Revista HUPE, Rio de Janeiro, v. 13, n. 2, p. 75-82, abr./ago. 2014.

DIAS, B. B.; MOTA, R. S.; GÊNOVA, T. C.; TAMBORELLI, V.; PEREIRA, V. V.; PUCCINI, P. T. Aplicação da Escala de Equilíbrio de Berg para verificação do equilíbrio de idosos em diferentes fases do envelhecimento. $R B C E H$, Passo Fundo, v. 6, n. 2, p. 213-224, maio/ago. 2009.
ELLENBECKER, T. S. Reabilitação dos ligamentos do joelho. Barueri: Manole, 2002.

ESQUENAZI, D.; SILVA, S. B.; GUIMARÃES, M. A. A fisiopatologia do envelhecimento humano e sua relação com a propensão a quedas em indivíduos idosos. Revista HUPE, Rio de Janeiro, v. 13, n. 2, p. 45-56, abr./jun. 2014.

FIGLIOLINO, J. A. M.; MORAIS, T. B.; BERBEL, A. M.; DAL CORSO, S. Análise da influência do exercício físico em idosos com relação a equilíbrio, marcha e atividade de vida diária. Rev. Bras. Geriatr. Gerontol., Rio de Janeiro, v. 12, n. 2, p. 227-238, 2009.

FIGUEIREDO, L. L.; PÍCOLI, T. S.; BORGES, A. P. O.; PATRIZZI, L. J. Análise do equilíbrio no processo de envelhecimento. Fisioter. Mov., Curitiba, v. 24, n. 3, p. 401417, jul./set. 2011.

FOLSTEIN, M. F.; FOLSTEIN, S. E.; MCHUGH, P. R. "Mini-mental state". A practical method for grading the cognitive state of patients for the clinician. J. Psychiatr. Res., Great Britain, v. 12, n. 1, p. 189-198, Nov. 1975.

FRANCIULLI, S. E.; RICCI, N. A.; LEMOS, N. D.; CORDEIRO, R. C.; GAZZOLA, J. M. A modalidade de assistência Centro-Dia Geriátrico: efeitos funcionais em seis meses de acompanhamento Multiprofissional. Ciênc. Saúde Coletiva, Rio de Janeiro, v. 12, n. 2, p. 373-380, mar./abr. 2007.

FREITAS, S. A.; CARVALHO, R. L.; VILAS BOAS, V. Controle postural em idosos: aspectos sensoriais e motores. Revista de Iniciação Científica da Universidade Vale do Rio Verde, Três Corações, v. 3, n. 2, p. 19-29, 2013.

GARDNER, M. M.; ROBERTSON, M. C.; CAMPBELL, A. J. Exercise in preventing falls and fall related injuries in older people: a review of randomised controlled trials. $\mathrm{Br}$. J. Sports Med., v. 34, n. 1, p. 7-17, Feb. 2000.

GAUCHARD, G. C.; GANGLOFF, P.; JEANDEL, C.; PERRIN, P. P. Influence of regular proprioceptive and bioenergetic physical activities on balance control in elderly women. J. Gerontol., v. 58, n. 9, p. 846-850, Sept. 2003. 
KATZ, S.; AKPOM, C. A. A Medida da principal função sociobiológica. Int. J. Health Serv., v. 6 , n. 1, p. 493-507, 1976.

LANUEZ, F. V.; JACOB FILHO, W. Efeitos de dois programas de exercícios físicos nos determinantes de aptidão motora em idosos sedentários. Einstein, São Paulo, v. 6, n. 1, p. 76-81, 2008.

LINO, V. T. S.; PEREIRA, S. E. M.; CAMACHO, L. A. B.; RIBEIRO FILHO, S. T.; BUKSMAN, S. Adaptação transcultural da Escala de Independência em Atividades da Vida Diária (Escala de Katz). Cad. Saúde Pública, Rio de Janeiro, v. 24, n. 1, p. 103112, jan. 2008.

LOPES, M. N. S. S.; PASSERINI, C. G.; TRAVENSOLO, C. F. Eficácia de um protocolo fisioterapêutico para equilíbrio em idosos institucionalizados. Semina. Ciênc. Biol. Saúde, Londrina, v. 31, n. 2, p. 143-152, jul./ dez. 2010.

LUNDEBJERG, N. Guideline for prevention of falls in older persons. J. Am. Geriatr. Soc., v. 49, n. 5, p. 664-672, May 2001.

MACIEL, A. C. C.; GUERRA. R. O. Prevalência e fatores associados ao déficit de equilíbrio em idosos. Rev. Bras. Ciênc. Mov., Brasília, v. 13, n. 1, p. 37-44, 2005.

MIYAMOTO, S. T.; LOMBARDI, J.; BERG, K.O.; RAMOS, L.R.; NATOUR, J. Brazilian version of the Berg balance scale. Braz. J. Med. Biol. Res. Ribeirão Preto-SP, v. 37, n. 9, p. 1411-1421, 2004.

NASCIMENTO, L. C. G.; PATRIZZI, L. J.; OLIVEIRA, C. C. E. S. Efeito de quatro semanas de treinamento proprioceptivo no equilíbrio postural de idosos. Fisioter. Mov., Curitiba, v. 25, n. 2, p. 325-331, abr./jun. 2012.

OLIVEIRA, D. L. C.; GORETTI, L. C.; PEREIRA, L.S.M. O desempenho de idosos institucionalizados com alterações cognitivas em atividades de vida diária e mobilidade: estudo piloto. Rev. Bras. Fisioter., São Carlos, v. 10, n. 1, p. 91-96, jan./mar. 2006.

PAULA, F. L. Envelhecimento e quedas de idosos. Rio de Janeiro: Apicuri, 2010.
SILVA, T. O.; FREITAS, R. S.; MONTEIRO, M. R.; BORGES, S. M. Avaliação da capacidade física e quedas em idosos ativos e sedentários da comunidade. Rev. Soc. Bras. Clín. Méd., São Paulo, v. 8, n. 5, p. 392-398, set./out. 2010.

SOUTHIARD, V.; DAVE, M.; DAVIS, M. G.; BLANCO, J.; HOFFERBER, A. The multiple tasks test as a predictor of falls in older adults. Gait Posture, v. 22, n. 4, p. 351-355, Dec. 2005.

SOUZA, A. Propriocepção. Rio de Janeiro: MEDSI, 2004.

STEADMAN, J.; DONALDSON, N.; KALRA, $\mathrm{L}$. A randomized controlled trial of an enhanced balance training program to improve mobility and reduce falls in elderly patients. Jornal da American Geriatrics Society, v. 51, n. 6, p. 847-852, June 2003.

WHITNEY, S.; WRISLEY, D.; FURMAN, J. Concurrent validity of the Berg Balance Scale and the Dynamic Gait Index in people with vestibular dysfunction. Physiother. Res. Int., v. 8, n. 4, p. 178-86, 2003.

YOKOKAWA, Y.; KAI I.; USUI, Y.; KOSODA, F.; FURUTA, T.; KONAKA, K. Intervention study using a fall prevention program to prevent functional decline of old - old elderly in a rural community. Nihon Ronen Igakkai Zasshi, v. 40, n. 1, p. 47-52, Jan. 2003.

ZAMBALDI, P. A.; COSTA, T. A. B. N.; DINIZ, G. D. L. N.; SCALZO, P. L. Efeito de um treinamento de equilíbrio em um grupo de mulheres idosas da comunidade: estudo piloto de uma abordagem específica, não sistematizada e breve. Acta. Fisiátrica, São Paulo, v. 14, n. 1, p. 17-24, mar. 2007. 